Lemmon Mark, T (Orcid ID: 0000-0002-4504-5136)

Guzewich Scott, D. (Orcid ID: 0000-0003-1149-7385)

Vicente-Retortillo Alvaro (Orcid ID: 0000-0002-4553-7624)

Martinez German (Orcid ID: 0000-0001-5885-236X)

Smith Michael, D. (Orcid ID: 0000-0002-7601-1158)

Confidential manuscript submitted to Geophys. Res. L.

\title{
Large dust aerosol sizes seen during the 2018 Martian global dust event by the Curiosity rover
}

\author{
M. T. Lemmon ${ }^{1}$, S. D. Guzewich ${ }^{2}$, T. McConnochie ${ }^{3}$, A. de Vicente-Retortillo ${ }^{4}$, G. \\ Martinez $^{5}$, Michael D. Smith ${ }^{2}$ J. F. Bell III ${ }^{6}$, D. Wellington ${ }^{6}$, and S. Jacob
}

${ }^{1}$ Space Science Institute, College Station, TX, USA; ${ }^{2}$ NASA Goddard Spaceflight Center, Greenbelt MD, USA; ${ }^{3}$ Department of Astronomy, University of Maryland, College Park, MD, USA; ${ }^{4}$ College of Engineering, University of Michigan, Ann Arbor, MI, USA; ${ }^{5}$ Lunar and Planetary Institute, Houston, TX, USA; ${ }^{6}$ School of Earth and Space Exploration, Arizona State University, Tempe, Arizona, USA.

Corresponding author: Mark Lemmon (MLemmon@SpaceScience.org)

\section{Key Points:}

- The Curiosity rover observed dust aerosol size variations through the 2018 global dust event

- The average dust radius increased above $4 \mu \mathrm{m}$, more than double the largest sizes previously seen with Curiosity's instruments

- The observations demonstrate the lifting and regional-scale transport of dust significantly larger than typical dust aerosol

This is the author manuscript accepted for publication and has undergone full peer review but has not been through the copyediting, typesetting, pagination and proofreading process, which may lead to differences between this version and the Version of Record. Please cite this article as doi: 10.1029/2019GL084407

This article is protected by copyright. All rights reserved. 


\begin{abstract}
Mars' atmosphere typically supports dust aerosol with an effective radius near $1.5 \mu \mathrm{m}$, varying from $\sim 1 \mu \mathrm{m}$ during low dust times near northern summer solstice to $\sim 2 \mu \mathrm{m}$ during higher dust times in southern spring and summer. After global dust events, size variations outside this range have not previously been observed. We report on imaging and spectral observations by the Curiosity rover through the 2018 global dust event. These observations show that the dust effective radius was seasonally normal prior to the local onset of increased opacity, increased rapidly above $4 \mu \mathrm{m}$ with increasing opacity, remained above $3 \mu \mathrm{m}$ over a period of $\sim 50$ Martian solar days, then returned to seasonal values before the opacity did so. This demonstrates lifting and regional scale transport of a dust population $\sim 3$ times the size of typical dust aerosol.
\end{abstract}

\title{
Plain Language Summary
}

During the global dust storm of 2018, the Curiosity rover measured the variation of atmospheric dust over time. During the onset of the dust storm, typical Martian dust was enhanced by much larger particles that were freshly lifted off the surface in distant storms and then carried to the rover site at Gale crater. The larger dust particles persisted for weeks, but fell out of the atmosphere faster than the typical dust as normal conditions were restored.

\section{Introduction}

Radiative balance in the martian atmosphere is dependent upon the time- and spatially-variable distribution of dust within it. Airborne dust has a dominant impact on the modern climate through the absorption and scattering of shortwave radiation and absorption and emission of longwave radiation [e.g., Gierasch and Goody, 1972]. The composition, shape, and size of the dust particles govern their interaction with radiation in the atmosphere [e.g., Wolff et al., 2006; Smith, 2008; Lemmon et al., 2015]. These dust particle properties can be retrieved directly from observations of the martian atmosphere (specifically the wavelength-dependent way it scatters, absorbs, and/or emits radiation) and have been done so by a broad set of spacecraft missions and instruments from both the surface and orbit [see Dlugach et al., 2003, for a review]: Mariner 9 [Pang et al., 1976; Toon et al., 1977], Viking orbiters [Clancy and Lee, 1991; Ockert-Bell et al., 1997], Viking landers [Pollack et al., 1995], Pathfinder [Tomasko et al., 1999], the Mars Exploration Rovers [Lemmon et al., 2004], Mars Global Surveyor Thermal Emission Spectrometer [Wolff and Clancy, 2003; Clancy et al., 2003; Wolff et al., 2006; Clancy et al., 2010], Mars Express [Rannou et al., 2006; Määttänen et al., 2013], the Mars Reconnaissance Orbiter [Wolff et al., 2009; Guzewich et al., 2014], and the Mars Science Laboratory Curiosity rover [Vicente-Retortillo et al., 2017; McConnochie et al., 2018; Chen-Chen et al., 2019]. With a few exceptions, most of these results have found that dust particle size varies by approximately $50 \%$ around a canonical value of $\sim 1.5 \mu \mathrm{m}$ in effective radius $\left(\mathrm{r}_{\mathrm{eff}}\right.$, a term of convenience in describing the particle size distribution, e.g. Deirmendjian [1964]). Occasional evidence of smaller particles [e.g., Rannou et al., 2006] and larger particles, particularly in global dust events [Clancy et al., 2010], has also been observed. However, limited systematic studies of dust size 
evolution after such events show mixed results: no evidence for a change in size after the 1971 storm [Toon et al., 1977], and a slow decay of particles size from about 1.5 to about 1.1 after the 2007 event [Vincendon et al. 2009].

The martian global dust event of 2018 (Mars Year 34 in the calendar of Clancy et al. [2000]) represented a rare opportunity to observe dust particles during an extreme event from the surface at low latitudes with a spacecraft unaffected by the loss of solar energy. The Mars Science Lander (MSL, or Curiosity rover) performed a dedicated science campaign to study the event, which raised the local dust optical depth from 0.6 to 8.5 despite a lack of dust lifting by storms at the site [Guzewich et al., 2019]. Curiosity's location in Gale Crater, just south of the equator, placed it nearer to the major centers of dust lifting than the Viking landers during the 1977/Mars Year 12 global dust events, while the Mars Exploration Rovers had to reduce or cease science operations during the global dust storm of 2007/Mars Year 28 due to the significant reduction in generated electricity by their solar panels.

In this work, we describe a series of independent retrievals of dust particle effective radius by the Curiosity rover's Mast Camera (Mastcam) and Chemistry and Camera (ChemCam) instruments during the 2018/Mars Year 34 global dust event.

\section{Data and models}

\subsection{Optical depth data}

Through the dust event, atmospheric optical depth was tracked via direct solar imaging with Mastcam [Guzewich, 2019]. Images were taken through 440- and 880-nm filters, reduced to radiance on sensor and integrated to flux. A standard Beer-Lambert-Bougher law, similar to Smith and Lemmon [1999] and Lemmon et al. [2015], produces normal optical depth. For several observations early in the event, the signal was weak-leading to a low signal-to-noise measurement - or non-existent. In order to supplement the standard solar filters, with their neutral-density-5 extinction [Bell et al. 2017; Malin et al. 2017], non-solar filters were employed for times of high path opacity. Several observations at transitional optical depths (expected path opacity of 9.6-11.5) were used to cross-calibrate the Mastcam R2 (447-nm) with R7 (440-nm ND5) and the L5 (867-nm) with L7 (880-nm ND5), while other observations used only the nonsolar filters. For this work, unlike for Guzewich et al. [2019], the 440-nm filters are of particular interest.

\subsection{Cross-sky imaging}

Mastcam cross-sky imaging surveys were specifically designed to retrieve dust particle size and shape information based on the angular dependence of solar radiation scattering by dust particles at several wavelengths. They involve a series of near-Sun images with Mastcam's L1 (527 nm), L2 $(445 \mathrm{~nm})$, and L4 (676 nm) filters, a series of images at constant elevation and increasing

azimuth (and hence, increasing scattering angle), and a series of images at varying elevations 
opposite the Sun, such that mid-range scattering angles were sampled at different elevations. Sky surveys also included a contemporaneous Mastcam opacity observation. Sky surveys were scheduled in the late afternoon, when the solar elevation angle was between $25-35^{\circ}$, on several sols. Images used in this study have been radiometrically calibrated as described by Bell et al. [2017]. Information on dust particle size primarily results from the change in $\mathrm{I} / \mathrm{F}$ as a function of scattering angle within $\sim 40^{\circ}$ of the Sun. A discrete ordinates radiative transfer forward model [Smith and Wolff, 2014] was used to model the light scattering (directly, the sky brightness in I/F) using the sky survey image geometry, observed Mastcam atmospheric opacity, and a series of assumed dust particle effective radii (see Guzewich et al., 2014 for details on dust particle size distributions and effective variance). The results from the forward model were compared to the observations and the dust particle effective radius was determined based on the lowest chisquared fit to the observations.

\subsection{Systematic near-Sun imaging}

Before and through the dust event, most opacity sequences included near-Sun sky imaging. Others omitted the sky imaging to save time, or (e.g., for the images that included both solarfilter- and non-solar-filter-opacity) truncated the sky images to fit within mission-imposed limits on commands within $22^{\circ}$ of the Sun. Beginning with sol (Martian solar day) 487, such near-Sun images typically included L2 for 445-nm radiometry to constrain particle size via diffractiondominated measurements. Images capturing scattering angles of $\sim 5-20^{\circ}$ were aimed such that the camera baffle prevented direct sunlight from hitting the optics. Calibrated radiances in this range were reduced to I/F using the L2/R2 response ratio measured via sky imaging [Bell et al. 2017] and solar fluxes measured directly with R2 contemporaneously with standard opacity measurements, resulting in a consistent, relative calibration.

The determined I/F as a function of scattering and zenith angle was modeled with DISORT (Discrete Ordinates Radiative Transfer, Stamnes et al. [1988] to determine aerosol size: For a fixed size-distribution variance of 0.3 , the cross-section weighted mean radius $\left(\mathrm{r}_{\text {eff }}\right)$ was adjusted to find a minimum in chi-squared. The process was repeated for several particle shape models (the reference model was the MSL fit from Bell et al. [2018]; spheres; Tomasko et al. [1999] particles, corrected for size error noted by Wolff et al. [2009]; and two permutations of the Tomasko model tuned to large and small irregular particles with variance of 0.8 ) to capture the uncertainty in scattering behavior at non-sampled scattering angles. The uncertainties in $r_{\text {eff }}$ were frequently dominated by model-to-model variability of $\sim 0.4 \mu \mathrm{m}$, rather than by individual fits or imaging statistics. The main conclusion of this work, a sudden rise in particle size by a factor of $>2$, was seen for each shape model. Figure 1 shows the average I/F as a function of scattering angle (in $0.1^{\circ}$ bins) for several measurements across the event. Note that the pre- and post-event fits are of high quality; the fits during the event are poorer. There is some indication that during the event, the size distribution was considerably broader and the variance of 0.3 was too narrow. In addition, for the largest aerosols modeled, the Legendre expansion of the phase function and the number of streams were inadequate. Nonetheless, $10-20^{\circ}$ scattering angles 
probe diffraction from near the 2-3 $\mu \mathrm{m}$ part of the size distribution at $445-\mathrm{nm}$ wavelengths, so the presence of an unusually large component to the size distribution is clearly seen. Future work will examine more aspects of constraints on the size distribution.

Except for the dust event, interannual variability was limited (Fig. 2), with repeatable variation around an average size of $1.47 \mu \mathrm{m}$. Near $\mathrm{L}_{\mathrm{s}} 135$, minimum sizes were $0.8-0.9 \mu \mathrm{m}$ in MY 32-34; the minimum was broadest in MY 32 and narrowest in MY 33. Highs near $\mathrm{L}_{\mathrm{s}} 220$ and 340 were seen in both MY 32 and 33, with identical behavior from $L_{s} 150$ until 60. For MY 32, the retrieved sizes were similar to those reported by Chen-Chen et al. [2019] from 650-nm diffraction observations, by Vicente-Retortillo et al. [2017] from ultraviolet fluxes, and spectral determination of opacity by McConnochie et al. [2018]. In each case, the seasonal pattern of variation is the same as seen in this work. For MY 33, there is more divergence near $L_{s} 90$, which may be related to differing treatments of water ice. Complete agreement across these methods is not expected given poor knowledge of how the shape affects the scattering behavior.

\subsection{Sky spectral data}

Chemcam passive-mode spectra of the sky were acquired on several sols to retrieve dust aerosol size from spectral variations. Observations were performed and aerosol size was modeled as described in McConnochie et al. [2018], although minor modifications to the aerosol property fitting procedure were required for cases where the Mastcam-derived optical depth exceeded 2.5 As McConnochie et al. [2018] describe, for Chemcam passive-mode sky observations aerosol size was constrained by the spectrum of the ratio of high- and low-elevation-angle pointing positions. Those two pointing positions $\left(65^{\circ}\right.$ and $20^{\circ}$ elevations angles) had azimuths chosen to have as nearly as possible matching predicted sky brightness at $720 \mathrm{~nm}$, and thus they often had similar scattering angles. A single aerosol shape model (equant cylinders) was modelled for $\mathrm{r}_{\text {eff }} \leq 8 \mu \mathrm{m}$.

When the Mastcam-derived optical depth exceeded 2.5 the standard fitting procedure described by McConnochie et al. [2018] produced poor fits. This occurred because at high dust opacity the ratio spectra become more sensitive to uncertainty in the Mastcam-derived opacity (which the Chemcam aerosol models were constrained to match), and become less sensitive to ice aerosol abundance. To solve this problem, we performed downhill-simplex fitting for a single parameter - dust aerosol $r_{\text {eff }}$ - with fixed assumptions for ice aerosol opacity, ice aerosol particle size, and percent-error in the Mastcam-derived opacity. We repeated the fitting procedure for a matrix of reasonable values of these fixed assumptions, adopting the dust $r_{\text {eff }}$ from the fit with the lowest chi-square value as our preferred solution and adopting a $95 \%$ confidence interval based on the chi-square values of all of the fits (Fig. 3).

The range of values considered for these fixed assumptions was 1-3 $\mu \mathrm{m}$ for ice particle effective radius (with no significant effect), 0 to 1.5 for $880 \mathrm{~nm}$ ice aerosol opacity, and $\pm 5 \%$ for $880 \mathrm{~nm}$ optical depth error. For the one case where Chemcam sky spectra coincided with low signal levels in the Mastcam optical depth ( $\left.\operatorname{sol} 2085, \mathrm{~L}_{\mathrm{s}}=195.4\right)$, we used a $\pm 10 \%$ optical depth error. 
The upper limit of 1.5 for the ice aerosol contribution is $50 \%$ larger than the largest ice aerosol opacities that have been inferred for the Mars equatorial region [Wilson et al., 2007] and so represents an extreme upper limit for a global dust storm scenario. Adopting a much lower upper limit for an ice aerosol contribution would have changed our estimate of the dust aerosol $\mathrm{r}_{\text {eff }}$ significantly in only one case: sol $2118\left(\mathrm{~L}_{\mathrm{s}}=215.8^{\circ}\right)$, for which lower maximum ice contribution leads to higher dust particle size but much poorer fits to the ratio spectrum data. While poorer fits seem to suggest a large contribution from ice aerosol, it is more likely that our adopted dust aerosol shape model is inadequate and the apparent ice aerosol only compensates for the incorrect dust shape.

We tested the grid of fixed assumptions on all of the ChemCam sky spectra cases with optical depth $<2.5$, but we found no case in which it produced results different from our standard procedures. For these low optical depth cases we give confidence intervals of $\pm 0.1 \mu \mathrm{m}$ based on the sensitivity testing of McConnochie et al. [2018]. We also adopt the $\pm 0.1 \mu \mathrm{m}$ confidence interval for high optical depth cases when it would be wider than given by the grid of fixed assumptions.

\subsection{Ultraviolet fluxes}

Rover Environmental Monitoring Station (REMS) ultraviolet (UV) observations have also been used to analyze variations in dust aerosol particle size using a methodology that relies on the output of the REMS UV sensor when the view of the Sun is blocked by the mast of the rover. The particle size was retrieved by comparing the measured photocurrents to those simulated using a Monte Carlo radiative transfer model and the empirically derived angular response and field of view of the sensor and have previously been reported by Vicente-Retortillo et al. [2017]. Retrievals from REMS UV measurements are in good agreement with those from Mastcam observations during a typical annual cycle (see Fig. 2). However, the methodology shows a loss of sensitivity for large particles. However, REMS UV fluxes indicated a sudden increase in dust particle size during the onset of the global dust storm, with $\mathrm{r}_{\mathrm{eff}}$ of $\sim 1.5 \mu \mathrm{m}$ on sol $2060, \sim 1.8 \mu \mathrm{m}$ on sol 2077, and significantly above $2 \mu \mathrm{m}$ on sol 2096.

\section{Results}

\subsection{Optical depth color ratio}

The Angstrom exponent $(\alpha)$ is defined such that opacity $(\tau)$ varies with wavelength $(\lambda)$ as $\left(\tau / \tau_{0}\right)=$ $\left(\lambda_{0} / \lambda\right)^{\alpha}$. The Angstrom exponent approaches four for molecular scattering, and has small positive or negative values for large particles. Mars dust opacity typically increases slightly with wavelength in the visible range [Smith and Lemmon, 1999], indicating a small, negative exponent. Smith and Lemmon [1999] used the Tomasko et al. [1999] model to derive a wavelength dependence of opacity that corresponds to an angstrom exponent of -0.16 , but added a small-particle scattering term to fit the actual opacity profile (which sometimes fell from blue 
to red, but increased from red to near-infrared). Lemmon et al. [2015] used 880- to 440-nm flux ratios to derive an opacity ratio with reduced systematic error compared to the opacity ratio; these correspond to Angstrom exponents of -0.047 and -0.036 for Spirit and Opportunity, respectively, but were likely influenced by spectral leaks.

Through sol 1450, the observed flux ratios corresponded to an average exponent of -0.082 \pm 0.011 , where the uncertainty is driven by the absolute flux uncertainty rather than the statistics of individual points (Fig. 2b). Little variation was seen, compared to the typical measurement uncertainties of 0.06, except additional variability around $\mathrm{L}_{\mathrm{S}} 100-130$ and a percent-scale secular change around sol 1450 to 1600 that was likely related to changes in transmission into the optics caused by external contamination. Over sols 1450-1600, the changes do not look like other changes in the same season, and correspond to a time when debris contaminated the MastCam baffles. Season for season, the angstrom exponent appears to be higher by $\sim 0.042$ after this, which is likely to be an artifact of differing camera response (the mean $\alpha$ in Fig. $2 b$ adjusts to 0.40 to compensate for this).

During the dust event, the exponent became larger, suggestive of larger particles (Fig. 4). The values shown in Fig. 4 differ from those shown in Fig. 2 in being corrected for the change in $\alpha$. The uncertainty is lower during the event, due to the long path length of dust in all observations. However, the exponent is only weakly dependent on size within the few- $\mu \mathrm{m}$ size range, so size cannot be retrieved independently of possible other changes. Nonetheless, the Angstrom exponent provides evidence for a change in the aerosol population during the event that is consistent with an increase in dust size.

\subsection{Aerosol size variations over seasons and the dust event}

During Mars Year 34, $\mathrm{r}_{\text {eff }}$ fell from $\sim 1.7 \mu \mathrm{m}$ to $\sim 1.3 \mu \mathrm{m}$ at the southern winter solstice, fell to $\sim 1$ $\mu \mathrm{m}$ and recovered to $\sim 1.4 \mu \mathrm{m}$ by sol 2000 and $\sim 1.5 \mu \mathrm{m}$ by the southern spring equinox immediately before the dust event. The opacity rose from 0.79 on sol 2075 to 2.11 on sol 2081, while $\mathrm{r}_{\text {eff }}$ increased to $1.89 \pm 0.09 \mu \mathrm{m}$. Figure 4 shows two peaks in particle size: a sharp spike at sols 2084-2087, with best fits above $7 \mu \mathrm{m}$ and lower limits above $6 \mu \mathrm{m}$; and a broader peak around sol 2110-2120, reaching $6 \mu \mathrm{m}$. Dust size remained above $3 \mu \mathrm{m}$ until sol $\sim 2130$, and fell essentially monotonically to seasonal values by sols 2140-2160. Smith et al. [2019] report a double peak in boundary-layer opacity that they attribute to an influx of dust into the crater. Their result aligns with the size peaks, suggesting that large particles may be confined low.

Sky survey results confirm the pattern, with sparser temporal sampling, but a wider range of angles to constrain the scattering. The last pre-storm size was $1.4 \mu \mathrm{m}$ on sol $2065 ; 1.65 \pm 0.1$ and $1.75 \pm 0.1 \mu \mathrm{m}$ sizes were seen on sols 2077 and 2080 as opacity began to rise; and there was a peak at $4.3 \pm 0.7 \mu \mathrm{m}$ on sol 2097 followed by a decline to $1.7 \pm 0.1 \mu \mathrm{m}$ by sol 2162 .

Spectral measurements with ChemCam showed particles sizes of 0.9-1.1 over Ls 144-170; climbing to $7.25 \mu \mathrm{m}$ (lower limit, $6 \mu \mathrm{m}$ ) on sol 2085 and 2.3-4.0 $\mu \mathrm{m}$ on sols 2096 and 2118; and 
falling to $1.81 \pm 0.1 \mu \mathrm{m}$ on 2140 . With the sol 2084 near-Sun images, the sol 2085 ChemCam measurement shows the early spike in dust size. These may be outliers; however, the angstrom exponent also shows a small but significant extreme on these sols.

For $\sim 4 \mu \mathrm{m}$, the sedimentation rate is expected to be $\sim 1 \mathrm{~km} / \mathrm{sol}$ in the bottom scale height [Kahre et al. 2008], implying a 10-sol sedimentation timescale. This, with the variable opacity and dust size, suggest episodic resupply over sols 2084-2133 as dust lifted in the last 1-10 sols was transported to Gale crater.

Mariner 9 observations after the 1971 dust event showed the fallout of dust occurring without a change in the relative abundance of large and small particles, indicating turbulent eddies dominated Stokes-Cunningham fallout [Toon et al. 1977]. However, the Mariner 9 observations began approximately 2 months after the peak of the dust storm, and thus correspond to a period when our measurements of size had nearly returned to normal. Mars Express observations after the 2007 event showed a slow decline, but with particles always below $1.6 \mu \mathrm{m}$. As those measurements preferentially sample the upper atmosphere, it is possible that large particles existed at low altitude or that the 2007 event was different. For the Viking Lander 1 and Opportunity events of 1976-77 and 2007, Pollack et al. [1979] and Lemmon et al. [2015] reported faster fallout rates shortly after the peak of the event, followed by a slower exponential decay later. While not unique, this could correspond to a shift from sedimentation of large particles to slower diffusion of all particles. While a change in rate was not reported in the MSL opacity data [Guzewich et al. 2019], the observability is complicated by the Ls 252-276 data gap due to a rover anomaly.

\section{Conclusions}

Curiosity rover instruments were used to measure aerosol size before and during the 2018 global dust event. Prior to the event, an extensive set of new size measurements agrees with previously reported patterns of a size minimum near $1 \mu \mathrm{m}$ during early northern summer and two size maxima in southern spring and summer near 1.8 and $2 \mu \mathrm{m}$.

During the first sols of the dust event at Gale crater, the average dust radius increased rapidly from seasonal values near $1.5 \mu \mathrm{m}$ to over $4 \mu \mathrm{m}$ immediately after the opacity peak. The radius remained $>3 \mu \mathrm{m}$ for $\sim 50$ sols before declining to seasonal values by 90 sols after the event was first observed locally. A double peak in size correlates with a double peak in boundary layer opacity, suggesting the possibility the large particles are confined low and difficult to detect from orbit.

The observed size increase demonstrates that large particles (compared to typical Martian dust aerosol) were lifted in the dust storms and transported over regional scales to Gale crater. The mean particle size approximately tripled, then returned to normal by about sol 2165, 10-40 sols before opacity reached its seasonal value (which was obscured by a rover anomaly). This happened while opacity was falling approximately as fast as it had in the aftermath of the Viking 
Lander 1 and Opportunity dust events of 1976 and 2007 [Guzewich et al. 2019], suggesting that the observed change in aerosol size distribution may be a typical feature of such storms.

\section{Acknowledgments}

This work was funded by NASA's Mars Exploration Program through the Mastcam, MAHLI, and MARDI instrument team and a MSL Participating Scientist grant to S. Guzewich. This research was made possible through the hard work of the MSL development and operations team, and especially the Mastcam operations group. A portion of the research was carried out at the Jet Propulsion Laboratory, California Institute of Technology, under a contract with the National Aeronautics and Space Administration. MSL data are freely available on the Planetary Data System within 6 months after receipt on Earth. Data used to make the figures are included in the supporting information.

\section{References}

Bell, J. F., A. Godber, S. McNair, M.A. Caplinger, J.N. Maki, M.T. Lemmon, J. Van Beek, M.C. Malin, D. Wellington, K.M. Kinch, M.B. Madsen, C. Hardgrove, M.A. Ravine, E. Jensen, D. Harker, R.B. Anderson, K.E. Herkenhoff, R.V. Morris and E. Cisneros. The Mars Science Laboratory Curiosity Rover Mast Camera (Mastcam) Instruments: Pre-Flight and In-Flight Calibration, Validation, and Data Archiving (2017). Earth and Space Science 4, 396-452. DOI: 10.1002/2016EA000219.

Chen-Chen, H., S. Pérez-Hoyos, and A. Sánchez-Lavega (2019). Dust particle size and optical depth on Mars Retrieved by the MSL navigation cameras. Icarus 319, 43-57. DOI:

10.1016/j.icarus.2018.09.010.

Clancy, R. T., and S. W. Lee (1991), A new look at dust and clouds in the Mars atmosphere: Analysis of emission-phase-function sequences from global Viking IRTM observations, Icarus, 93, 135-158.

Clancy, R. T., Sandor, B. J., Wolff, M. J., Christensen, P. R., Smith, M. D., Pearl, J. C., Conrath, B. J., Wilson, R. J. (2000). An intercomparison of ground---based millimeter, M GS TES, and Viking atmospheric temperature measurements: Seasonal and interannual variability of temperatures and dust loading in the global Mars atmosphere, Journal of Geophysical Research, 105 (E4), 9553-9572.

Clancy, R.T., Wolff, M.J., Christensen, P.R. (2003). Mars aerosol studies with the MGS TES emission phase function observations: Optical depths, particle sizes, and ice cloud types versus latitude and solar longitude. J. of Geophys. Res. 108, doi: 10.1029/2003JE002058.

Clancy, R. T., Wolff, M. J., Whitney, B. A., Cantor, B. A., Smith, M. D., McConnochie, T. H. (2010). Extension of atmospheric dust loading to high altitudes during the 2001 Mars dust storm: MGS TES limb observations. Icarus, 207 (1), 98-109. 
Deirmendjian, D. (1964). Scattering and polarization properties of water clouds and hazes in the visible and infrared. Applied Optics 3, 187. DOI: 10.1364/AO.3.000187.

Dlugach, Z. M., Korablev, O. I., Morozhenko, A. V., Moroz, V. I., Petrova, E. V., Rodin, A. V. (2003). Physical Properties of Dust in the Martian Atmosphere: Analysis of Contradictions and Possible Ways of Their Resolution, Solar System Research, 37 (1), 1-19.

Gierasch, P. J., Goody, R. M. (1972). The effect of dust on the temperature of the Martian atmosphere, Journal of Atmospheric Science, 29, 400- 402.

Guzewich, S. D., Smith, M. D., and Wolff, M. J. ( 2014), The vertical distribution of Martian aerosol particle size, J. Geophys. Res. Planets, 119, 2694- 2708, doi:10.1002/2014JE004704."

Guzewich, S.D., M. Lemmon, C.L. Smith, G. Martinez, A. Vicente-Retortillo, C.E. Newman, M. Baker, C. Campbell, B. Cooper, J. Gomez-Elvira, A.-M. Harri, D. Hassler, F.J. Martin-Torres, T. McConnochie, J.E. Moores, H. Kahanpaa, A. Khayat, M.I. Richardson, M.D. Smith, R. Sullivan, M. de la Torre Juarez, A.R. Vasavada, D. Viudez-Moreiras, C. Zeitlin, and M.P. Zorzano Mier (2019). Mars Science Laboratory observations of the 2018/Mars year 34 global dust storm. Geophys. Res. Lett., 46, 71-79. DOI: 10.1029/2018GL080839.

Kahre, M. A., J. L. Hollingsworth, R. M. Haberle, and J. R. Murphy (2008). Investigations of the variability of dust particle sizes in the martian atmosphere using the NASA Ames General Circulation Model. Volume 195, 576-597. DOI: 10.1016/j.icarus.2008.01.023.

Lemmon, M.T., M.J. Wolff, M.D. Smith, R.T. Clancy, D. Banfield, G.A. Landis, A. Ghosh, P.H. Smith, N. Spanovich, B. Whitney, P. Whelley, R. Greeley, S. Thompson, J.F. Bell III, S.W. Squyres (2004). Atmospheric Imaging Results from the Mars Exploration Rovers: Spirit and Opportunity. Science 306, 1753-1756, doi: 10.1126/science.1104474.

Lemmon, M.T., M.J. Wolff, J.F. Bell III, M.D. Smith, B. Cantor, and P.H. Smith (2015). Dust aerosol, clouds, and the atmospheric optical depth record over 5 Mars years of the Mars Exploration Rover mission. Icarus 251, 96-111. DOI: 10.1016/j.icarus.2014.03.029.

Määttänen, A., Listowski, C., Montmessin, F., Maltagliati, L., Reberac, A., Joly, L., Bertaux, J.L. (2013). A complete climatology of the aerosol vertical distribution on Mars from MEx/SPICAM UV solar occultations. Icarus, Volume 223, 892-941. DOI: 10.1016/j.icarus.2012.12.001.

Malin, M.C., M.A. Ravine, M.A. Caplinger, F.T. Ghaemi, J.A. Schaffner, J.N. Maki, J.F. Bell III, J.F. Cameron, W.E. Dietrich, K.S. Edgett, L.J. Edwards, J.B. Garvin, B. Hallet, K.E. Herkenhoff, E. Heydari, L.C. Kah, M.T. Lemmon, M.E. Minitti, T.S. Olson, T.J. Parker, S.K. Rowland, J. Schieber, R. Sletten, R.J. Sullivan, D.Y. Sumner, R.A. Yingst, B.M. Duston, S. McNair, and E.H. Jensen (2017). The Mars Science Laboratory (MSL) Mast cameras and Descent imager: I. Investigation and instrument descriptions. Earth and Space Science 4, 506539. DOI: 10.1002/2016EA000252 
Confidential manuscript submitted to Geophys. Res. L.

McConnochie, T.H., M.D. Smith, M.J. Wolff, S. Bender, M.T. Lemmon, R.C. Wiens, S. Maurice, O. Gasnault, J. LaSue, P.-Y. Meslin, A.-M. Harri, M. Genzer, O. Kemppinen, G.M. Martínez, L. DeFlores, D. Blaney, J.R. Johnson, and J.F. Bell III (2018). Retrieval of water vapor column abundance from ChemCam passive sky spectroscopy. Icarus 307, 294-326. https://doi.org/10.1016/j.icarus.2017.10.043.

Ockert-Bell, M. E., Bell, J. F., Pollack, J. B., McKay, C. P., Forget, F. (1997). Absorption and scattering properties of the Martian dust in the solar wavelengths, Journal of Geophysical Research, 102 (E4), 9039-9050.

Pang, K., J. M. Ajello, C. W. Hord, and W.G. Egan (1976). Complex Refractive Index of Martian Dust: Mariner 9 Ultraviolet Observations. Icarus, Volume 27, 55-67. DOI: 10.1016/0019-1035(76)90184-6.

Pollack, J. B., Colburn, D. S., Flasar, F. M., Kahn, R., Carlston, C. E., \& Pidek, D. G. (1979). Properties and effects of dust particles suspended in the Martian atmosphere. Journal of Geophysical Research, 84(B6), 2929-2945. https://doi.org/10.1029/JB084iB06p02929.

Pollack, J.B., Ockert-Bell, M.E., Shepard, M.K., (1995). Viking Lander image analysis of martian atmospheric dust. J. Geophys. Res. 100 (E3), 5235-5250. DOI: 10.1029/94JE02640.

Rannou, P., Perrier, S., Bertaux, J.-L., Montmessin, F., Korablev, O., Reberac, A. (2006). Dust and cloud detection at the Mars limb with UV scattered sunlight with SPICAM, Journal of Geophysical Research, 111 (E9), DOI: 10.1029/2006JE002693.

Smith, C.L., J.E. Moores, M. Lemmon, S.D. Guzewich, C.A. Moore, D. Ellison, and A.S.J. Khayat (2019). Visibility and Line-Of-Sight Extinction Estimates in Gale Crater during the 2018/MY34 Global Dust Storm. Geophys. Res. Lett., in press. DOI: 10.1029/2019GL083788.

Smith, P.H., and M.T. Lemmon (1999). Opacity of the Mars atmosphere measured by the Imager for Mars Pathfinder. J. Geophys. Res., 104, 8975-8985, doi: 10.1029/1998JE900017.

Smith, M. D., \& Wolff, M. J. (2014). Dust aerosol particle size and shape using MER Navcam and Pancam sky imaging, presented at the $5^{\text {th }}$ International Workshop on the Mars Atmosphere: Modelling and Observations, Oxford, UK, January, 2014.

Smith, M. D., 2008. Spacecraft Observations of the Martian Atmosphere, Annual Review of Earth and Planetary Sciences, 36, 191-219.

Stamnes, K., S-C. Tsay, W. Wiscombe, and K. Jayaweera (1988). Numerically stable algorithm for discrete-ordinate-method radiative transfer in multiple scattering and emitting layered media. Appl. Opt., 27(12):2502-2509.

Tomasko, M.G., L.R. Doose, M.T. Lemmon, P.H. Smith, and E. Wegryn 1999. Properties of dust in the martian atmosphere from the Imager for Mars Pathfinder. J. Geophys. Res., 104, 8987-9007, doi: 10.1029/1998JE900016. 
Toon, O. B., Pollack, J. B., Sagan, C. (1977). Physical properties of the particles composing the Martian dust storm of 1971---1972, Icarus, 30, 663 -696.

Vicente-Retortillo, Á., G. Martínez, N. Rennó, M.T. Lemmon, and M. de la Torre Juarez (2017). Determination of dust aerosol particle size at Gale Crater using REMS UVS and Mastcam measurements. Geophys. Res. L., 44. doi: 10.1002/2017GL072589.

Vincendon, M., Y. Langevin, F. Poulet, A. Pommerol, M. Wolff, J.-P. Bibring, B. Gondet, and D. Jouglet (2009). Yearly and seasonal variations of low albedo surfaces on Mars in the OMEGA/MEx dataset: Constraints on aerosols properties and dust deposits. Icarus 200, 395-405. DOI: 10.1016/j.icarus.2008.12.012.

Wilson,R. J., G. A. Neumann, and M. D. Smith (2007). Diurnal variation and radiative influence of Martian water ice clouds Geophys. Res. Lett. 34, 2710. DOI: 10.1029/2006GL027976.

Wolff, M. J., and R. T. Clancy (2003), Constraints on the size of Martian aerosols from thermal emission spectrometer observations, J. Geophys. Res., 108(E9), 5097,

doi:10.1029/2003JE002057.

Wolff, M.J., M.D. Smith, R.T. Clancy, N. Spanovich, B.A. Whitney, M.T. Lemmon, J. Bandfield, D. Banfield, A. Ghosh, G. Landis, P. Christensen, J.F. Bell III, and S. Squyres (2006). Atmospheric results from Mars Global Surveyor overflights of the Mars Exploration Rovers. J. Geophys. Res. 111, E12S17, doi: 10.1029/2006JE002786.

Wolff, M.J., Smith, M.D., Clancy, R.T., Arvidson, R., Kahre, M., Seelos, F., Murchie, S., Savijarvi, H. (2009). Wavelength dependence of dust aerosol single scattering albedo as observed by the Compact Reconnaissance Imaging Spectrometer. J. of Geophys. Res. 114, DOI: 10.1029/2009JE003350.

\section{Figure captions}

Figure 1. The variation of radiance with scattering angle. From the top, the data (black lines and gray 1- $\sigma$ confidence intervals) for sol 2144 (magenta fits), 2080 (red fits), 2170 (green fits), and 2115 (blue fits) are shown. Deviations from smooth curves are caused by corners of the image that have systematic departures from average zenith angles.

Figure 2. Dust properties with time. (A) Retrieved dust $\mathrm{r}_{\text {eff }}$ for MY 31 (blue), 32 (black), 33 (red) and 34 (green) is shown: circles, this work; triangles, Vicente-Retortillo et al. [2017]; diamonds, McConnochie et al. [2018]; and squares, Chen-Chen et al. [2019]. (B) 440-nm optical depth (blue diamonds) and retrieved dust $r_{\text {eff }}$ (gray circles, every third error bar) is shown. At the top, Mars year numbers indicate $\mathrm{L}_{\mathrm{S}}$ (solar longitude) 0 , or northern spring equinox, and additional $\mathrm{L}_{\mathrm{S}}$ values are noted. (C) 440- to 880-nm Angstrom exponent is shown. Light gray, dashed lines indicate mean values of (b) $r_{\text {eff }}$ and (c) Angstrom exponent.

Figure 3. The best model fit (heavy black line) to the continuum ratio spectrum (black points) is shown for sols 2085, 2096, 2118, and 2140. The light-weight lines represent models identical to 
the best fit except for an alternative dust aerosol particle $r_{\text {eff }}$ which is given in the legend. The error bars were obtained by scaling an assumed constant fractional uncertainty in order to obtain a reduced- $\chi 2$ of 1 for the best fit.

Figure 4. Dust properties around the dust event. (A) Size measurements are from cross-sky surveys (red hexagons); near-Sun images (gray circles); sky passive spectra (green diamonds); and UV fluxes (magenta triangles). For context, size measurements are shown from near-Sun images in MY 32 (light green circles) and 33 (light blue circles), as are 440-nm optical depth (blue diamonds). (B) 440- to 880-nm Angstrom exponent are shown. 


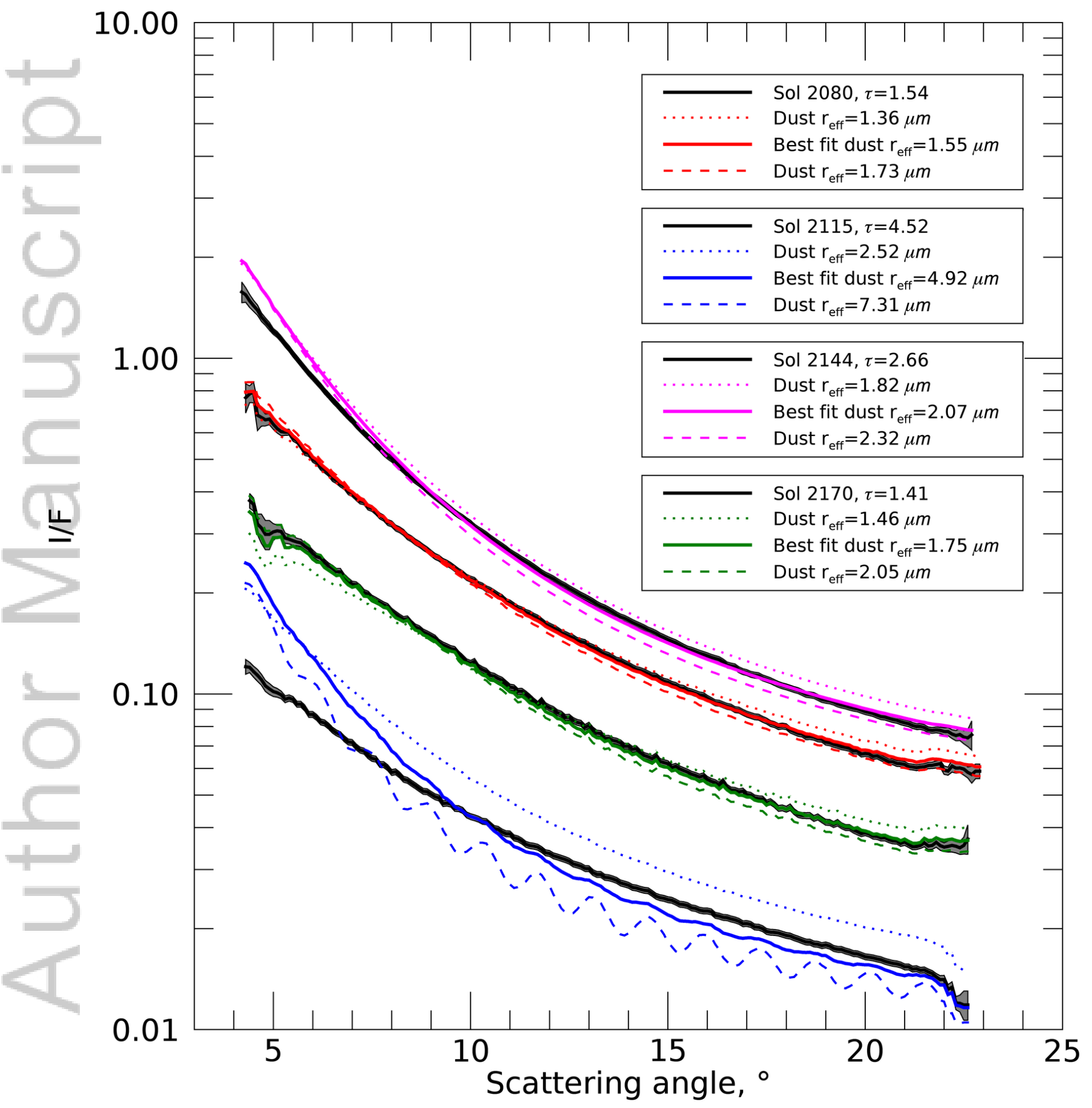




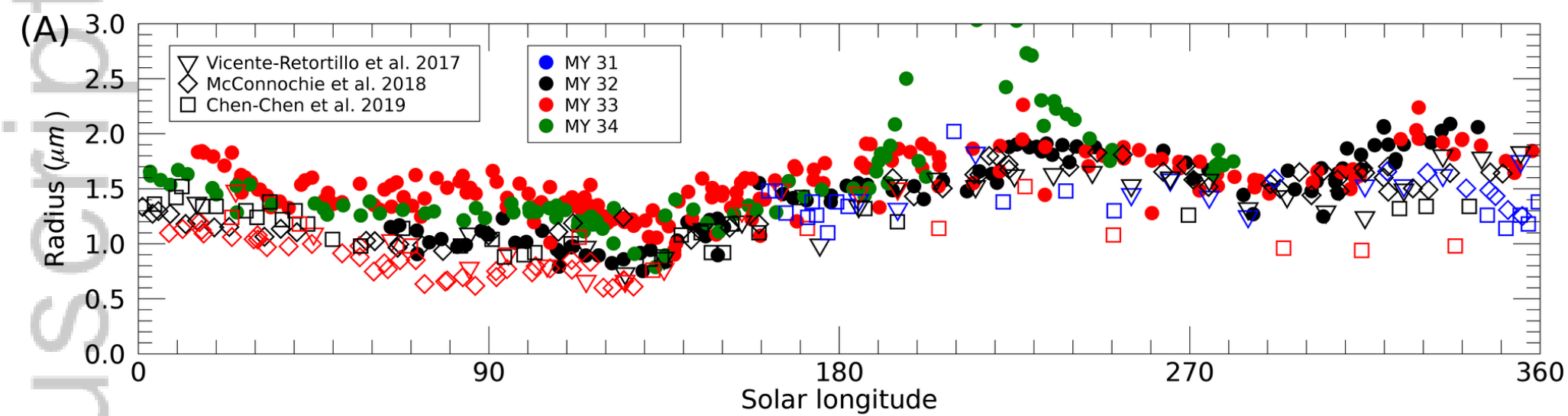

(B)

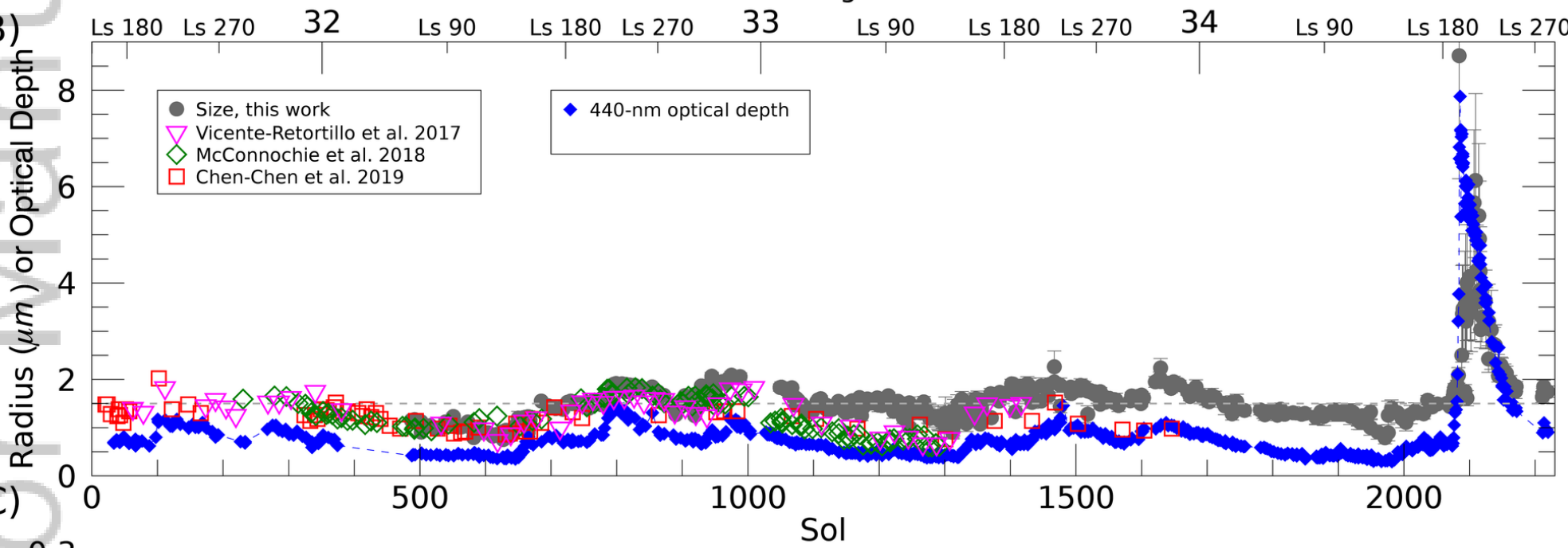

(C)

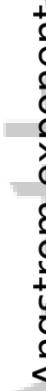

0.3

0.2

0.1

$-0.0$

0.1
0

1
3
0

E

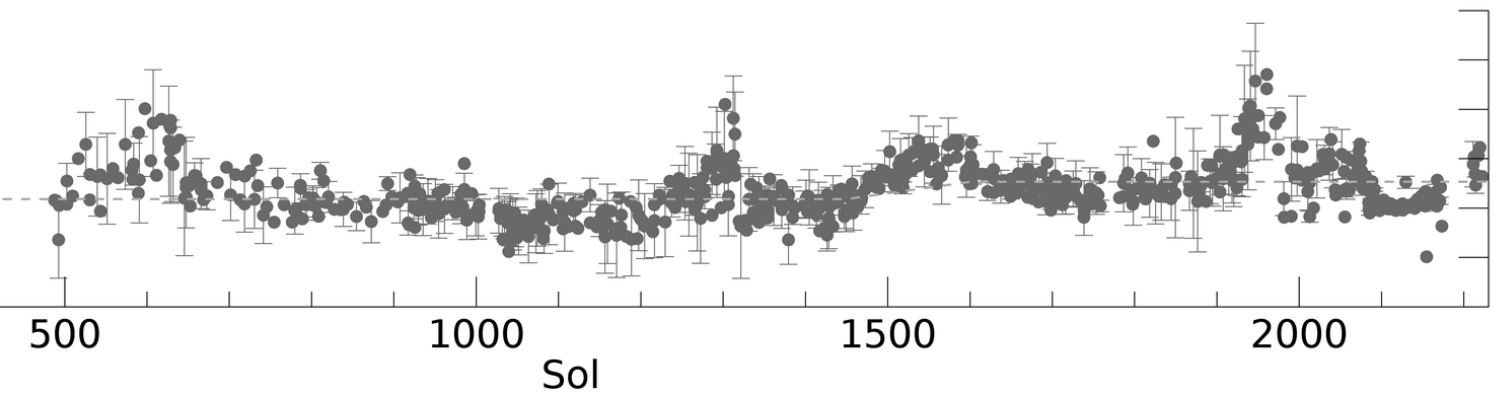

2019GL084407-f02-Z-.png

This article is protected by copyright. All rights reserved. 

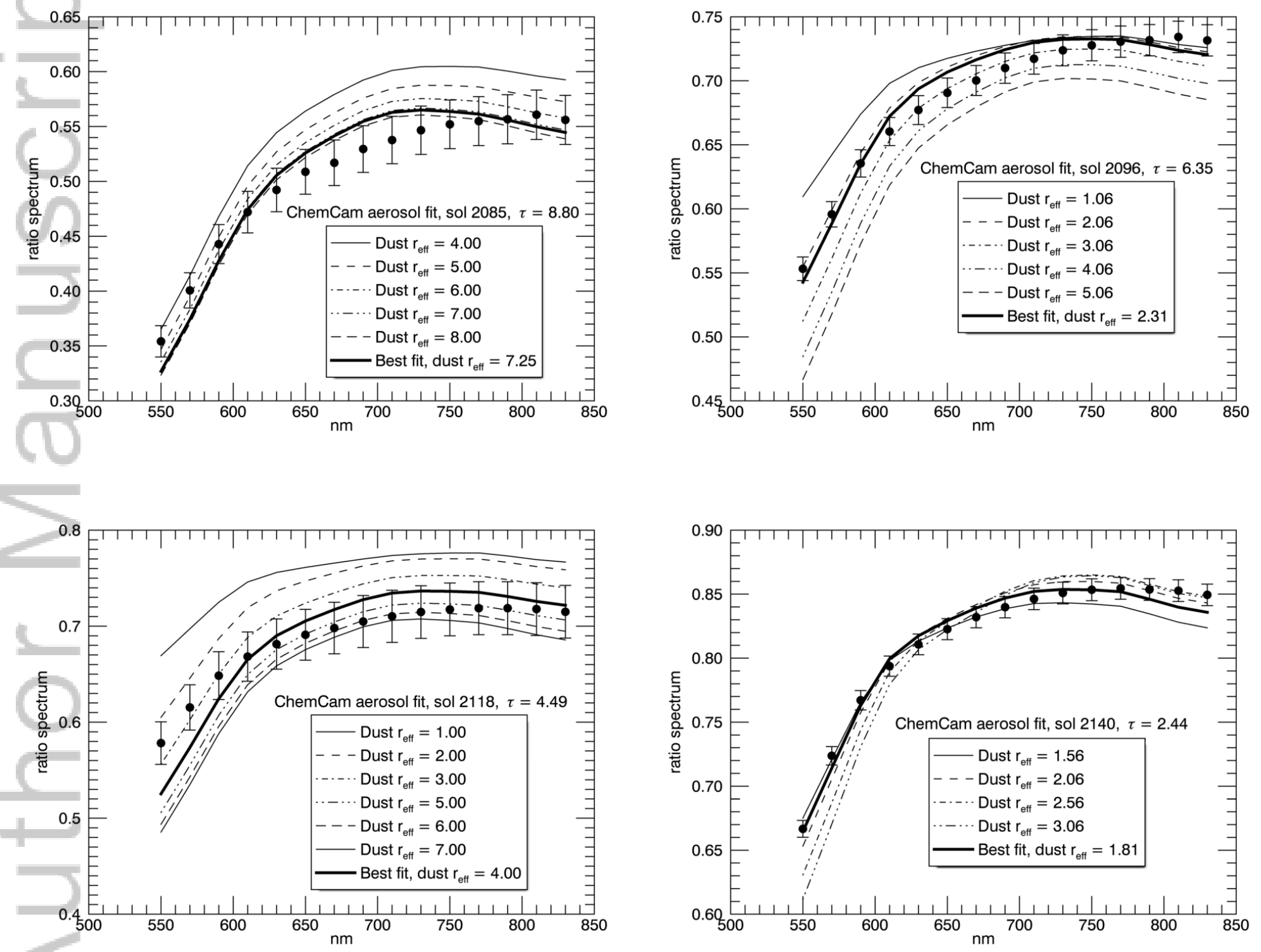

2019GL084407-f03-z-.png

This article is protected by copyright. All rights reserved. 
